\title{
BMJ Open Effects of crowding in the emergency department on the diagnosis and management of suspected acute coronary syndrome using rapid algorithms: an observational study
}

Kiril M Stoyanov, ${ }^{1}$ Moritz Biener, ${ }^{1}$ Hauke Hund, ${ }^{1,2}$ Matthias Mueller-Hennessen, ${ }^{1}$ Mehrshad Vafaie, ${ }^{1}$ Hugo A Katus, ${ }^{1}$ Evangelos Giannitsis (D) ${ }^{1}$

To cite: Stoyanov KM, Biener M, Hund $\mathrm{H}$, et al. Effects of crowding in the emergency department on the diagnosis and management of suspected acute coronary syndrome using rapid algorithms: an observational study. BMJ Open 2020;10:e041757. doi:10.1136/ bmjopen-2020-041757

- Prepublication history and additional material for this paper are available online. To view these files, please visit the journal online (http://dx.doi. org/10.1136/bmjopen-2020041757).

Received 16 June 2020 Revised 28 July 2020 Accepted 30 July 2020

D) Check for updates

(C) Author(s) (or their employer(s)) 2020. Re-use permitted under CC BY-NC. No commercial re-use. See rights and permissions. Published by BMJ.

${ }^{1}$ Department of Cardiology, Angiology and Pulmonology, Heidelberg University Hospital, Heidelberg, Germany

${ }^{2}$ Faculty of Informatics, Heilbronn University of Applied Sciences, Heilbronn, Germany

Correspondence to Professor Evangelos Giannitsis; Evangelos.Giannitsis@med.uniheidelberg.de

\section{ABSTRACT}

Objectives Fast diagnostic algorithms using highsensitivity troponin (hsTn) in suspected acute coronary syndrome (ACS) are regarded as beneficial to expedite diagnosis and safe discharge of patients in crowded emergency departments (ED). This study investigates the effects of crowding on process times related to the diagnostic protocol itself or other time delays, and outcomes.

Design Prospective single-centre observational study. Setting ED (Germany).

Participants Final study population of 2525 consecutive patients with suspected ACS within 12 months, after exclusion of patients with ST-elevation myocardial infarction, missing blood samples, referral from other hospitals or repeated visits.

Interventions Use of fast algorithms as per 2015 European Society of Cardiology guidelines.

Main outcome measures Crowding was defined as mismatch between patient numbers and monitoring capacities, or mean physician time per case, categorised as normal, high and very high crowding. Outcome measures were length of ED stay, direct discharge from ED, laboratory turn around times (TAT), utilisation of fast algorithms, absolute and relative non-laboratory time, as well as mortality.

Results Crowding was associated with increased length of ED stay (3.75-4.89 hours, $p<0.001)$. While median TAT of the first hsTnT increased $(53-57 \mathrm{~min}, \mathrm{p}<0.001)$, total TAT of serial hsTnT did not increase significantly with higher crowding $(p=0.170)$. Lower utilisation of fast algorithms $(p=0.009)$ and increase of additional hsTnT measurements after diagnosis $(p=0.001)$ were observed in higher crowding. Most importantly, crowding was significantly associated with prolonged absolute $(p<0.001)$, and particularly relative non-laboratory time $(63.3 \%$ $71.3 \%, p<0.001)$. However, there was no significant effect of crowding on mortality, even after adjustment for relevant clinical variables.

Conclusions Process times, and particularly nonlaboratory times, are prolonged in a crowded ED diminishing some positive effects of fast diagnostic algorithms in suspected ACS. Higher crowding levels were

\section{Strengths and limitations of this study}

- This is the first large observational study on crowding in the emergency department using rapid diagnostic algorithms in suspected acute coronary syndrome, showing the association of crowding with process times related to the diagnostic protocol itself and other time delays, as well as patient outcomes.

- All consecutive patients with suspected acute coronary syndrome were included, covering a broad spectrum of symptoms, including dyspnoea and other atypical symptoms.

- The study was carried out in a single experienced centre, thus in settings with different clinical expertise results may differ.

- The definition of crowding is hospital-specific and has not been externally validated, while process times depend also on factors beyond the biomarkerbased protocol.

not significantly associated with higher all-cause mortality rates.

Trial registration number NCT03111862.

\section{INTRODUCTION}

There is a growing body of evidence that emergency department (ED) crowding is linked to adverse patient outcomes, patient satisfaction and decrease in quality of care. ${ }^{1-3}$ Bernstein $e t$ $a t^{4}$ studied the effects of ED crowding on clinically oriented outcomes using the Englishlanguage literature for the years 1989-2007. Based on 369 studies, of which 41 were included, the authors reported increased risk of in-hospital mortality, longer times to treatment for patients with acute chest pain or pneumonia, and a higher probability of leaving the ED against medical advice, or without being seen at all. Notably, crowding 
was not associated with delays in reperfusion for patients with ST-elevation myocardial infarction (STEMI). ${ }^{4}$ In another population-based study on 13934542 patients in Canada, ${ }^{5}$ longer waiting times in the ED increased the short-term risk of mortality and admission to hospital, both in patients with high and low acuity except those who left ED without being seen.

EDs evaluating patients with suspected acute coronary syndrome (ACS) are particularly impacted by crowding, since chest pain represents the second most frequent reason for ED visits wordwide. In the USA, a total of 7.6 million patients visit an ED for chest pain and another 3.4 million for shortness of breath according to the National Hospital Ambulatory Medical Care Survey in $2016 .{ }^{6}$ Based on an overview on 58 studies, only $14 \%$ (IQR 10\%-20\%) of these patients receive a final diagnosis of myocardial infarction (MI). ${ }^{7}$ The problem is further fuelled by a temporal trend of increasing visits for chest pain reported in a National survey in England from 1998 to 2010, while rates of typical angina and confirmed MI remained stable or slightly decreased during the same period. ${ }^{8}$ Newer analyses from England for the period from years 2008/2009 to 2017/2018 demonstrate that ED admissions for ACS remained stable. ${ }^{9}$ This finding is largely consistent with data from the USA demonstrating that ED admissions for ACS decreased by 15\% from 2006 to $2013 .{ }^{10}$ In the same period, emergency visit rates for chest pain increased by $31 \% .^{10}$

For patients presenting with suspected ACS, 2015 European Society of Cardiology (ESC) guidelines ${ }^{11}$ advocate new accelerated diagnostic protocols that enable an earlier diagnosis of MI using very low or very high highsensitivity troponin (hsTn) levels at presentation, or early retesting of hsTn after 1 or 2 hours. Faster protocols were associated with shorter times to diagnosis and patient disposition in several observational trials. ${ }^{12-14}$ To date, this is only partly supported by randomised controlled trials. ${ }^{15-18}$ In addition, fast protocols were shown to be associated with reduced length of ED stay and costs. ${ }^{19-21}$ Moreover, discharge of low-risk patients has been demonstrated to be safe after accurate rule-out, and is not causing an overutilisation of hospital resources. ${ }^{12} 1421$ Therefore, it is tempting to speculate that fast protocols are likely to be adopted in crowded EDs due to their positive effects on decongestion. On the other hand, crowding might have a negative impact on time-dependent processes that are critical with the use of fast protocols, an issue that has not been addressed appropriately, so far. In most observational trials, physicians were blinded to the results of the investigational product and management decisions were made at the discretion of the treating physician, presumably without the pressure to decongest a busy ED.

A major obstacle to the systematic evaluation of possible effects of crowding in the ED is that there is no agreement on the measures that should be used to quantify crowding. ${ }^{22-24}$ Common criteria address the mismatch between the number of patients seeking medical attention and the limited number of beds and medical staff.
Accordingly, variables to describe crowding include number of occupied beds, numbers of patients and numbers of physicians, severity of presentation, as well as numbers of patients leaving without being seen.

The aim of the present study was to evaluate the potential effects of increasing crowding in the ED on processes and times including blood sampling intervals, utilisation rates of fast vs standard diagnostic protocols, length of ED stay, as well as mortality in patients presenting with suspected ACS.

\section{METHODS \\ Patient population}

In a prospective single-centre study in the ED of the Department of Cardiology, Department of Cardiology, Angiology and Pulmonology, Heidelberg University Hospital, consecutive patients with suspected ACS between 1 July 2016 and 30 June 2017 were evaluated. Patient disposition, times related to management processes, and treatments were collected in a 6-month preimplementation period followed by a 6 -month implementation period.

During the implementation period, the ESC 0/1-hour algorithm was officially declared as the primary diagnostic strategy over the standard 0/3-hour algorithm in order to further accelerate diagnosis and disposition to admission or discharge. Details on the study population and intervention have been published earlier. ${ }^{25}$ Briefly, patients qualified for enrolment with a clinically suspected ACS, based on broad spectrum of symptoms including atypical symptoms and dyspnoea. Patients with following clinical presentation were not included: documented AV nodal re-entry tachycardia; presentation with acute heart failure due to already known structural heart disease (eg, dilated cardiomyopathy); confirmed primary pulmonary disease (eg, asthma, chronic obstructive pulmonary disease, lobar pneumonia) without suspected ACS; traumatic chest pain with preceded thorax injury; confirmed dysfunction or alarm of an implantable cardiac device; chronic haemodialysis. Patients were not excluded for severe chronic kidney disease, older age, chronic heart failure or atrial fibrillation. Patients were not included in the case of inappropriate command of English/German language or permanent residence in a foreign country.

We performed a retrospective analysis on the effects of crowding on the troponin-based diagnostic algorithms and the non-laboratory-related process times. Other outcomes considered include hospital admission, length of ED stay, all-cause mortality and process times. Therefore, exclusion criteria comprised following: (1) repeated presentations beyond the index admission ('frequent flyer'), as they would artificially expand the study cohort; (2) patients referred from other hospitals for early or primary percutaneous coronary intervention without receiving standard diagnostic work-up and (3) diagnostic set of hsTnT samples not available (eg, missing initial or consecutive blood sample), as they are required to calculate troponin-based protocol time. Patients with STEMI 


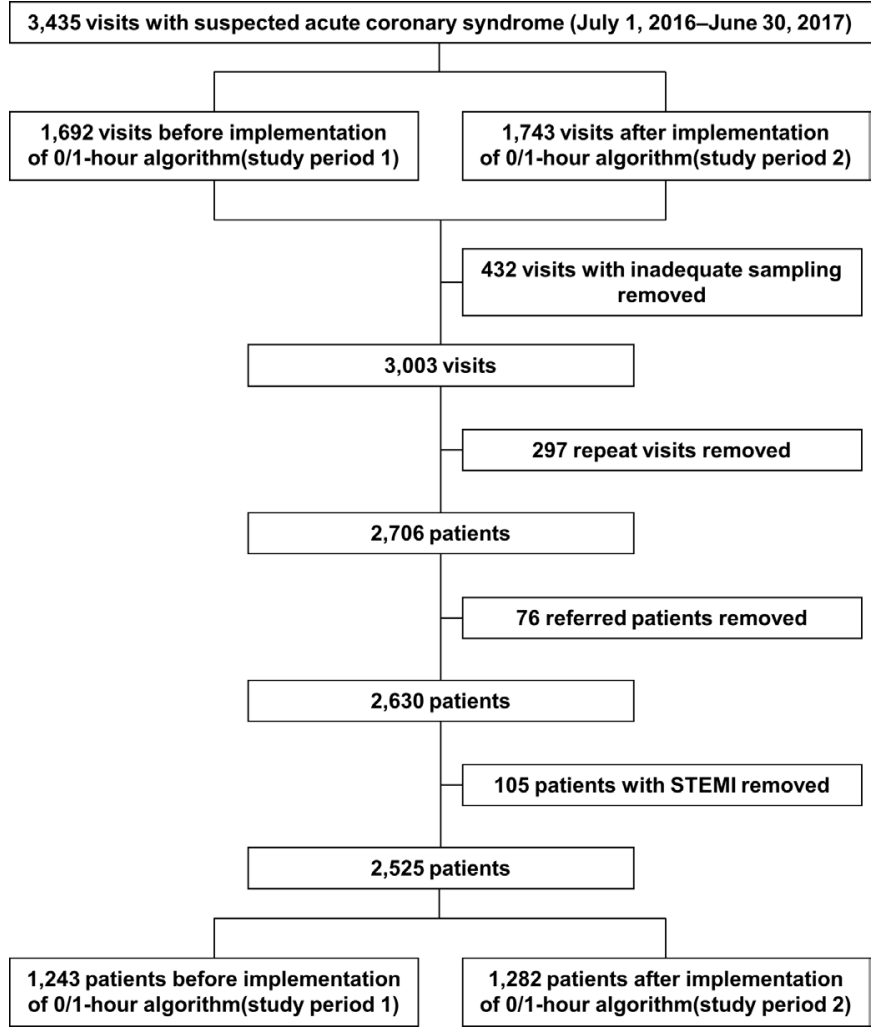

Figure 1 Patientinclusion flow diagram (reproduced with permission from SAGE publications ${ }^{25}$ ). STEMI, ST-elevation myocardial infarction.

were registered but were excluded for this analysis, since in these cases no biomarkers are required for the diagnosis. A consort diagram illustrates the screening process (figure 1).

On 1 January 2017, the ESC 0/1-hour algorithm was officially implemented. Prior to this date, every staff member in the ED had received training (formal education, posters and bedside cards) for the use and interpretation of the ESC 0/1-hour algorithm using hsTnT from 1 January 2017.

MI was diagnosed by the treating physicians based on all clinical information, using the diagnostic criteria of the third Universal MI definition. Patients were categorised using the validated biomarker criteria for classification into rule-out, observe or rule-in as proposed by the 2015 ESC guidelines on non-ST-elevation ACS. ${ }^{11}$ Levels of cardiac troponin were measured at presentation, after 1 or 3 hours, and thereafter as long as clinically indicated. All patients underwent a clinical assessment that included medical history, physical examination, 12-lead ECG, continuous ECG monitoring, pulse oximetry and standard blood tests. Results were reported on the electronic patient record and were communicated to the clinicians responsible for patients' care.

Patients received treatment at the discretion of the attending physician, and all decisions to admit or discharge, or on the need and timing of invasive coronary angiography were made based on available information during ED stay. The standard 12-lead ECG included routinely precordial leads $\mathrm{V}_{7}-\mathrm{V}_{9}$. The decision to discharge was at the discretion of the treating physician and comprised clinical judgement from individual risk variables, or the Global Registry of Acute Coronary Events (GRACE) score that was generated automatically by an electronic calculator embedded into the electronic file, and thus was accessible for all physicians. The GRACE score was included in the Cox regression model to identify independent predictive variables for all-cause mortality.

Follow-up for 12 months was accomplished using telephone, questionnaire, patient's hospital notes, the family physician's records and the municipal registry on vital status using hospital records, phone calls and questionnaires. For this observational study, informed consent of the individual patient was not required by the Ethics Committee of the Medical Faculty of Heidelberg.

\section{Description of the ED}

The ED of the Department of Cardiology, Department of Cardiology, Angiology and Pulmonology, Heidelberg University Hospital is the only specialised and regularly audited ED for cardiovascular emergencies covering a region of more than 500000 inhabitants. A total of 8500 patients per year are self-referred or admitted for evaluation and management of a broad spectrum of acute cardiovascular and respiratory emergencies including ACS, hypertensive emergencies, acute dyspnoea, syncope, alarm/ dysfunction/shock of an implantable cardiac device. Patients are ineligible for the ED after out-of-hospital cardiopulmonary resuscitation, or if haemodynamic ally/ respiratory unstable requiring circulatory support or mechanical ventilation. Patients with STEMI are transferred directly to the catheterisation laboratory and usually bypass the ED. The ED has access to coronary angiography, chest radiography, and CT with a 24/7 availability, and has full access to stress testing during working hours. The ED of the Department of Cardiology, Department of Cardiology, Angiology and Pulmonology, Heidelberg University Hospital in Heidelberg has a total of six monitoring beds plus three shared monitored beds that are used jointly with the general internal medicine ED. There is an option for six additional monitoring beds that are managed by the general internal medicine ED but can be occupied on demand only if available. The ED operates in a three shift system during workdays and a two shift system during weekends. The first shift is operated by two physicians, and the second and third shift by one physician. The ED is directed by a cardiologist who consults and supervises all decisions including indication and timing of coronary angiography, and qualification for admission or discharge. Attending physicians have experience on cardiovascular emergencies, are skilled in transthoracic and transoesophageal echocardiography, and are usually in training for internal medicine and cardiology specialisation.

\section{Definition of crowding}

Crowding was defined using a combination of two surrogate variables that reflect the shortage of monitoring 
capacities or effective physician time per patient. The first variable demonstrates the ratio between available monitoring beds and numbers of patients seeking simultaneously medical attendance. The second variable represents the total time spent per patient. Simultaneous monitoring was defined by the numbers of patients who were monitored concomitantly within the first $60 \mathrm{~min}$ after admission which represents the most labour-intensive time interval during diagnostic workup. Second, total time spent by a treating physician for an individual patient was defined as the time needed to collect information on symptoms and history, physical examination, blood draw(s), ECG reading, interpretation of diagnostic findings and drafting of discharge or referral report.

\begin{tabular}{|c|c|c|c|c|c|}
\hline & $\begin{array}{l}\text { All patients } \\
(n=2525)\end{array}$ & $\begin{array}{l}\text { Crowding level 1, } \\
\text { normal }(n=662)\end{array}$ & $\begin{array}{l}\text { Crowding level 2, high } \\
(n=1409)\end{array}$ & $\begin{array}{l}\text { Crowding level } 3 \text {, } \\
\text { very high }(n=454)\end{array}$ & $P$ value \\
\hline Age (years) & $64(18-101)$ & $64(18-100)$ & $65(18-100)$ & $62(18-101)$ & 0.386 \\
\hline Male, sex & $1465(58.0 \%)$ & $361(54.5 \%)$ & $852(60.5 \%)$ & $252(55.5 \%)$ & 0.019 \\
\hline \multicolumn{6}{|l|}{ Presenting symptom } \\
\hline Chest pain & $1164(46.1 \%)$ & $322(48.6 \%)$ & $645(45.8 \%)$ & 197 (43.4\%) & 0.21 \\
\hline Dyspnoea & $335(13.3 \%)$ & $91(13.7 \%)$ & 187 (13.3\%) & $57(12.6 \%)$ & 0.847 \\
\hline Atypical & $1021(40.4 \%)$ & 247 (37.3\%) & $575(40.8 \%)$ & $199(43.8 \%)$ & 0.085 \\
\hline Else or missing & $5(0.2 \%)$ & $2(0.3 \%)$ & $2(0.1 \%)$ & $1(0.2 \%)$ & 0.742 \\
\hline \multicolumn{6}{|l|}{ Final diagnoses } \\
\hline UA & $280(11.1 \%)$ & $48(7.3 \%)$ & $182(12.9 \%)$ & $50(11.0 \%)$ & $<0.001$ \\
\hline NSTEMI & $330(13.1 \%)$ & $108(16.3 \%)$ & 169 (12.0\%) & $53(11.7 \%)$ & 0.015 \\
\hline STEMI* & $133^{*}$ & $42^{*}$ & $66^{*}$ & $25^{\star}$ & 0.361 \\
\hline Non-ACS & 1915 (75.8\%) & $506(76.4 \%)$ & $1058(75.1 \%)$ & $351(77.3 \%)$ & 0.577 \\
\hline \multicolumn{6}{|l|}{ Risk factors } \\
\hline Arterial hypertension & $1652(65.4 \%)$ & $433(65.4 \%)$ & $943(66.9 \%)$ & $276(60.8 \%)$ & 0.058 \\
\hline Hypercholesterolaemia & $1135(45.0 \%)$ & $303(45.8 \%)$ & $633(44.9 \%)$ & $199(43.8 \%)$ & 0.815 \\
\hline Diabetes mellitus & $535(21.2 \%)$ & $151(22.8 \%)$ & 305 (21.6\%) & $79(17.4 \%)$ & 0.077 \\
\hline Active smoker & $551(21.8 \%)$ & 155 (23.4\%) & $287(20.4 \%)$ & $109(24.0 \%)$ & 0.135 \\
\hline Family history & $665(26.3 \%)$ & $180(27.2 \%)$ & $373(26.5 \%)$ & $112(24.7 \%)$ & 0.633 \\
\hline \multicolumn{6}{|l|}{ History } \\
\hline Coronary artery disease & $827(32.8 \%)$ & $222(33.5 \%)$ & $476(33.8 \%)$ & $129(28.4 \%)$ & 0.093 \\
\hline Myocardial infarction & $430(17.0 \%)$ & $131(19.8 \%)$ & $236(16.7 \%)$ & $63(13.9 \%)$ & 0.033 \\
\hline Coronary angiography & $1039(41.1 \%)$ & $272(41.1 \%)$ & 605 (42.9\%) & $162(35.7 \%)$ & 0.024 \\
\hline $\begin{array}{l}\text { Percutaneous coronary } \\
\text { intervention }\end{array}$ & $655(25.9 \%)$ & $179(27.0 \%)$ & $378(26.8 \%)$ & $98(21.6 \%)$ & 0.065 \\
\hline Coronary bypass surgery & $166(6.6 \%)$ & $40(6.0 \%)$ & $97(6.9 \%)$ & $29(6.4 \%)$ & 0.759 \\
\hline $\begin{array}{l}\text { High-sensitivity troponin } T \\
\text { at baseline(ng/L) }\end{array}$ & $10(<3-4085)$ & $9(<3-3379)$ & $10(<3-4085)$ & $10(<3-2776)$ & 0.065 \\
\hline
\end{tabular}

Renal function

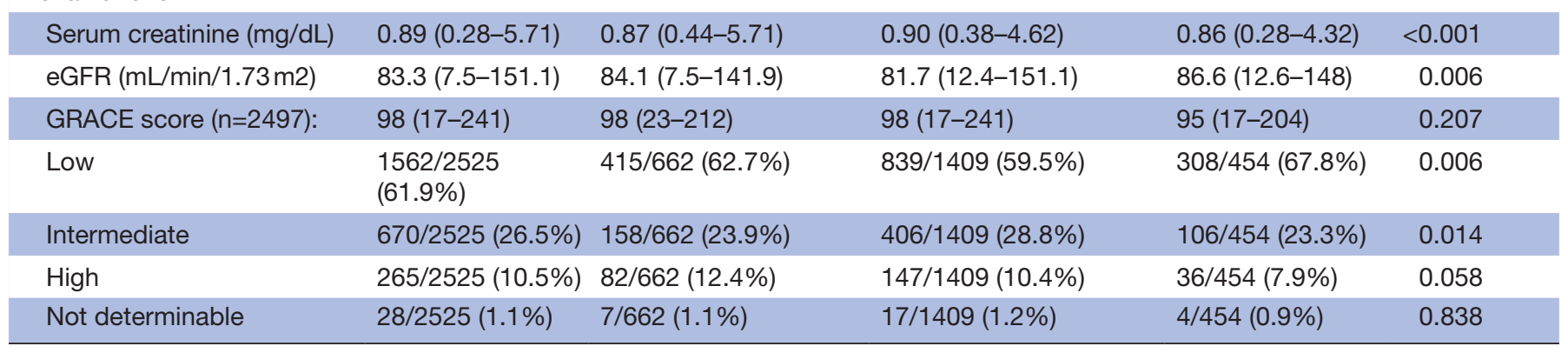

Data are median (minimum-maximum) or number of patients (\%).

*Patients with STEMI were registered but excluded for the analysis.

ACS, acute coronary syndrome; eGFR, estimated glomerular filtration rate; NSTEMI, non-ST-segment elevation myocardial infarction; STEMI, ST-segment elevation myocardial infarction; UA, unstable angina. 
The total time spent per patient was calculated as: total numbers of patients admitted per shift, divided by the number of physicians per shift. A period of 2 hours was subtracted per shift and per physician for general administrative work and for a rest break of $45 \mathrm{~min}$ per shift.

Accordingly, three categories of crowding were defined. Normal crowding level as concomitant monitoring of 5 or less patients, and a total physician time of $60 \mathrm{~min}$ or longer. High crowding level as concomitant monitoring of 6-9 patients, or a total physician time of 30-60 min per patient. Very high crowding level as concomitant monitoring of 10 or more patients, or total physician time of less than $30 \mathrm{~min}$ per patient.

\section{Management decisions, time stamps and critical time} intervals

Length of stay (LOS) in ED was registered as the exact time from administrative admission to administrative

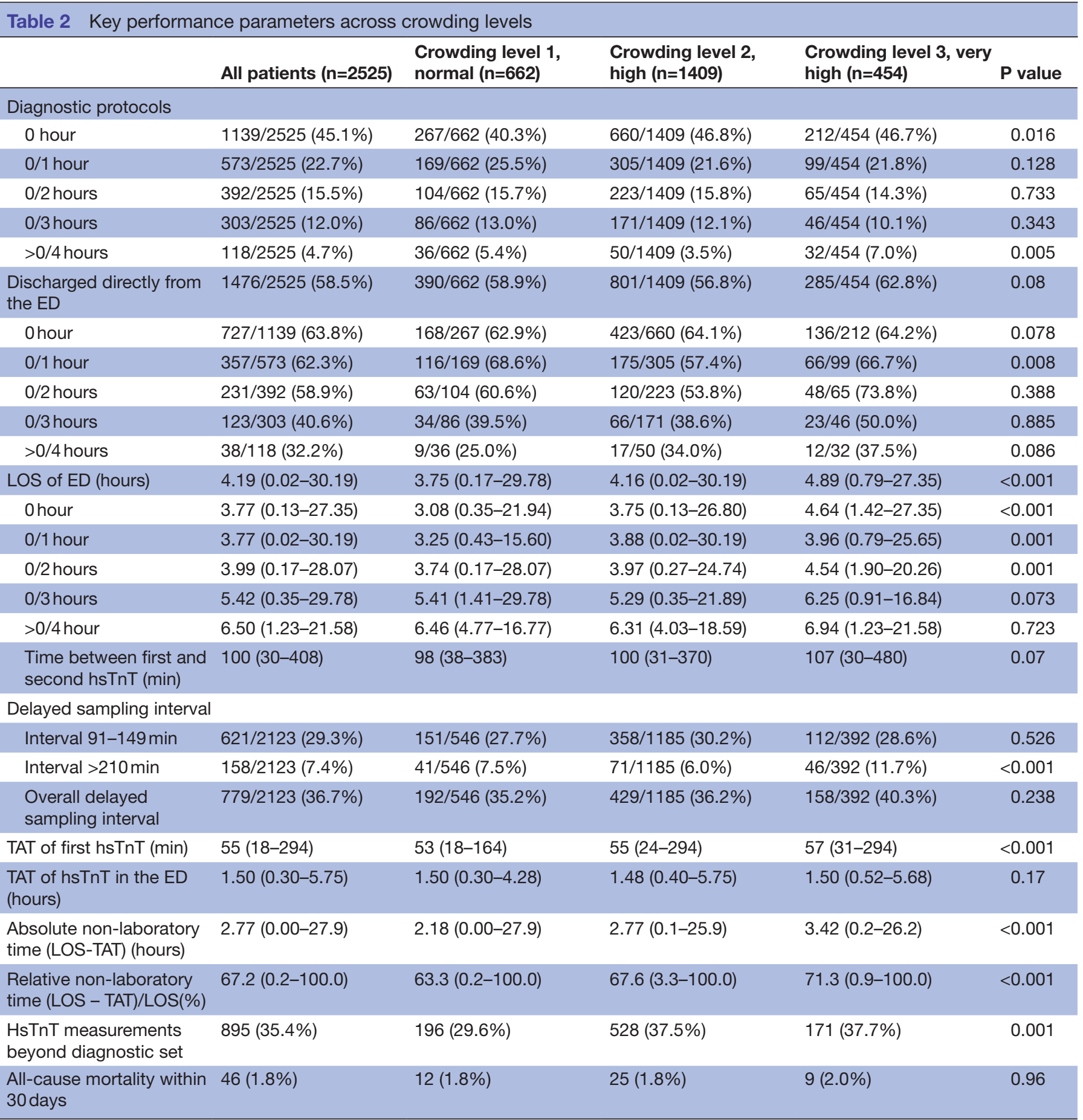

Data are median (minimum-maximum) or number of patients (\%).

ED, emergency department; hsTnT, high-sensitivity troponin T; LOS, length of stay; TAT, turn around time. 


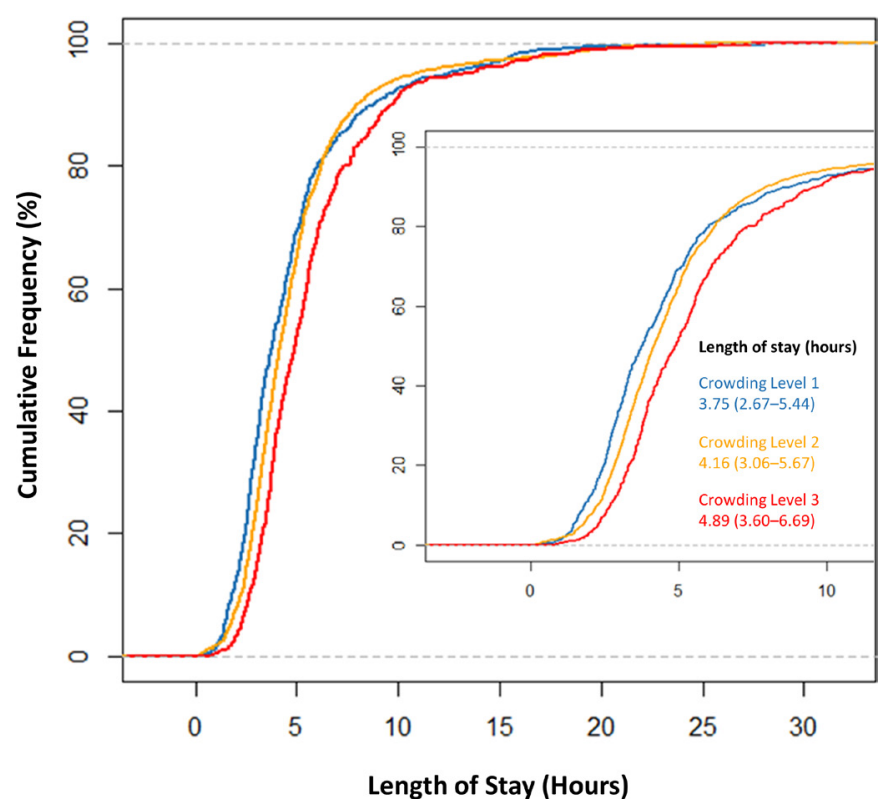

Figure 2 Cumulative distribution of length of stay (hours) in the emergency department by crowding level: normal (blue), high (yellow) and very high crowding (red).

discharge or transfer. The ED is connected to the central laboratory via a rapid pneumatic tube system, which allows transportation of blood samples to the central laboratory within $2 \mathrm{~min}$. On arrival in the central laboratory, blood samples are identified and registered automatically. Time stamps from the electronic laboratory reporting system were collected for the time to reporting of the results of each laboratory parameter. Turn around times (TAT) were defined as the time elapsed from registration of the blood sample in the central laboratory to final reporting of the value in the laboratory information system. Absolute non-laboratory time was defined as process time unrelated to algorithm and was calculated (LOS-total TAT). Relative non-laboratory time was defined as the percentage of process time unrelated to algorithm in relation to LOS.

\section{Follow-Up}

Follow-up was accomplished using telephone, questionnaire, patient's hospital notes, the family physician's records and the municipal registry.

\section{Statistical analysis}

Continuous variables were tested for normal distribution and were presented either as means with $95 \%$ CIs, or as medians with minimum and maximum. The normality of data distribution was assessed by the Kolmogorov-Smirnov test. Groups were compared using the $\chi^{2}$ test for categorical variables and Kruskal-Wallis test for continuous variables. Kaplan-Meier curves and the log-rank test were used

Table 3 Results of multiple regression for predictors of loge-transformed length of stay (minutes) in the emergency department

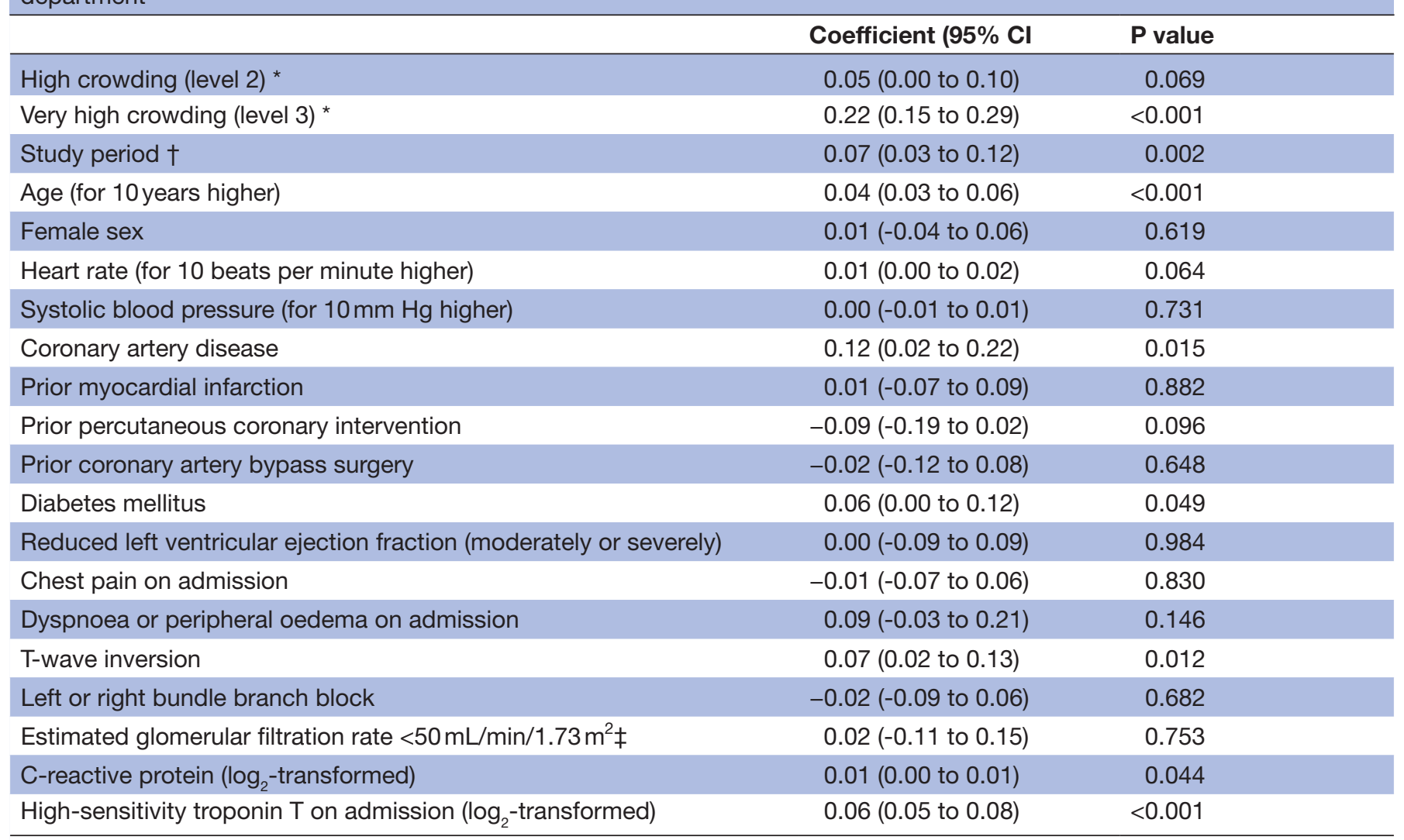

${ }^{\star}$ For the comparison with normal crowding (level 1).

†For the comparison of study period two with study period 1.

$\ddagger$ By modification of diet in renal disease equation. 
Table 4 Results of logistic regression for predictors of hospital admission

\begin{tabular}{|c|c|c|}
\hline & OR $(95 \% \mathrm{Cl})$ & $P$ value \\
\hline High crowding (level 2) * & $0.98(0.76$ to 1.26$)$ & 0.873 \\
\hline Very high crowding (level 3) * & $0.75(0.54$ to 1.05$)$ & 0.097 \\
\hline Age (for 10year higher) & $1.26(1.16$ to 1.37$)$ & $<0.001$ \\
\hline Female sex & $0.78(0.62$ to 0.98$)$ & 0.035 \\
\hline Systolic blood pressure (for $10 \mathrm{~mm} \mathrm{Hg}$ higher) & $0.95(0.91$ to 1.00$)$ & 0.050 \\
\hline Coronary artery disease & 1.68 (1.10 to 2.59$)$ & 0.017 \\
\hline Prior myocardial infarction & $0.87(0.62$ to 1.22$)$ & 0.429 \\
\hline Prior percutaneous coronary intervention & $0.91(0.58$ to 1.44$)$ & 0.697 \\
\hline Prior coronary artery bypass surgery & $0.99(0.64$ to 1.54$)$ & 0.958 \\
\hline Chest pain on admission & $1.44(1.05$ to 1.99$)$ & 0.023 \\
\hline Dyspnoea or peripheral oedema on admission & 3.38 (1.75 to 6.92$)$ & $<0.001$ \\
\hline T-wave inversion & $1.16(0.89$ to 1.51$)$ & 0.275 \\
\hline Left or right bundle branch block & 0.99 (0.70 to 1.40$)$ & 0.952 \\
\hline Estimated glomerular filtration rate $<50 \mathrm{~mL} / \mathrm{min} / 1.73 \mathrm{~m}^{2} \ddagger$ & $1.21(0.60$ to 2.66$)$ & 0.611 \\
\hline $\mathrm{C}$ reactive protein (for doubling in value) & 1.06 (1.03 to 1.09$)$ & $<0.001$ \\
\hline High-sensitivity troponin T on admission (for doubling in value) & $2.26(2.06$ to 2.51$)$ & $<0.001$ \\
\hline
\end{tabular}

${ }^{*}$ For the comparison with normal crowding (level 1).

†For the comparison of study period two with study period 1.

$\ddagger$ By modification of diet in renal disease equation.

to assess differences in outcomes across crowding levels. Multiple regression analysis was used to identify independent predictors for the continuous variable length of ED stay and for prediction of admission/discharge. Because distribution of the length of ED stay was skewed, we used the natural logarithm of length of ED stay. Multivariate Cox proportional hazards regression was performed to assess the impact of crowding level on all-cause mortality. As the present study is a secondary analysis of a study that had focused on the temporal effects of implementation of fast diagnostic protocols in suspected ACS, we calculated relative as well as absolute non-protocol-related process times and controlled for the study period (preimplementation vs postimplementation phase) and many other confounders. All regression analyses were adjusted for the study period. All hypothesis testing was two tailed and $p$ values $<0.05$ were considered statistically significant. All statistical analyses were performed using MedCalc V.11.1 and R V.3.6.1 (The R Foundation for Statistical Computing).

\section{Patient and public involvement}

Patients or public were not involved in the development of the study protocol.

\section{RESULTS}

A total of 2525 patients during a period of 12 months were enrolled. Baseline demographics of the entire cohort have been published earlier. ${ }^{25}$ Baseline parameters split by crowding levels are presented in table 1 . Prevalence of normal, high and very high crowding was $26.2 \%, 55.8 \%$ and $18.0 \%$. Key performance measures in relation to crowding levels are listed in table 2.

\section{Process times and crowding}

There was a significant effect of crowding on median length of ED stay with an increase from 3.75 to 4.89 hours $(p<0.001)$ (cumulative distribution on figure 2). LOS was disproportionately prolonged with all fast diagnostic protocols. There was a non-significant trend $(p=0.080)$ for higher discharge rates from ED with increasing crowding levels, presumably reflecting shortage of in-hospital bed capacities. Multiple regression analyses showed an independent association of LOS in the ED and hospital admission with very high crowding level (tables 3 and 4).

The median laboratory TAT of the first hsTnT increased significantly with increasing crowding levels, from 53 to $57 \mathrm{~min}(\mathrm{p}<0.001)$. In addition, there was a slight but insignificant prolongation of the blood sampling interval between the first and the second blood sample from 98 to $107 \mathrm{~min}(\mathrm{p}=0.070)$. However, the total TAT of serial 


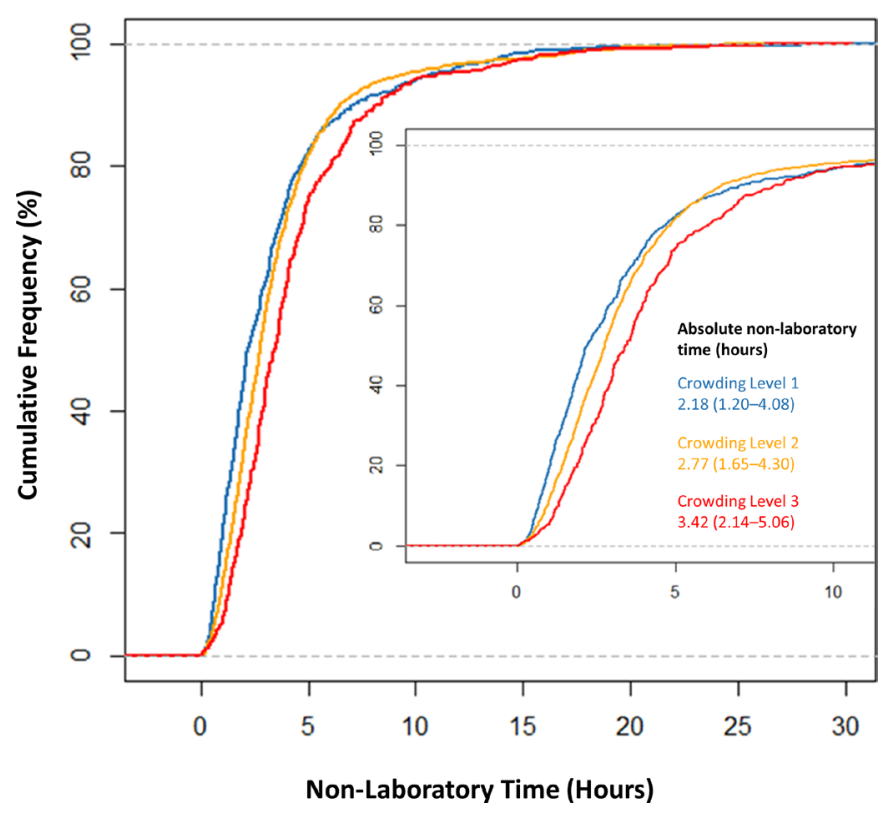

Figure 3 Cumulative distribution of the absolute nonlaboratory time (hours) by crowding level: normal (blue), high (yellow) and very high crowding (red).

hsTnT in the ED did not increase significantly with higher crowding levels $(p=0.170)$. The cumulative distributions of the TAT of the first hsTnT and the total TAT of hsTnT are shown on online supplemental figures S1 and S2.

Considering the small delays related directly to blood sampling and processing, the absolute non-laboratory time (LOS -TAT) increased significantly in higher crowding levels from 2.18 to 3.42 hours $(\mathrm{p}<0.001)$, and the relative non-laboratory time ((LOS - TAT)/LOS) from $63.3 \%$ to $71.3 \%(\mathrm{p}<0.001)$, suggesting a relevant impact of crowding on other process times including

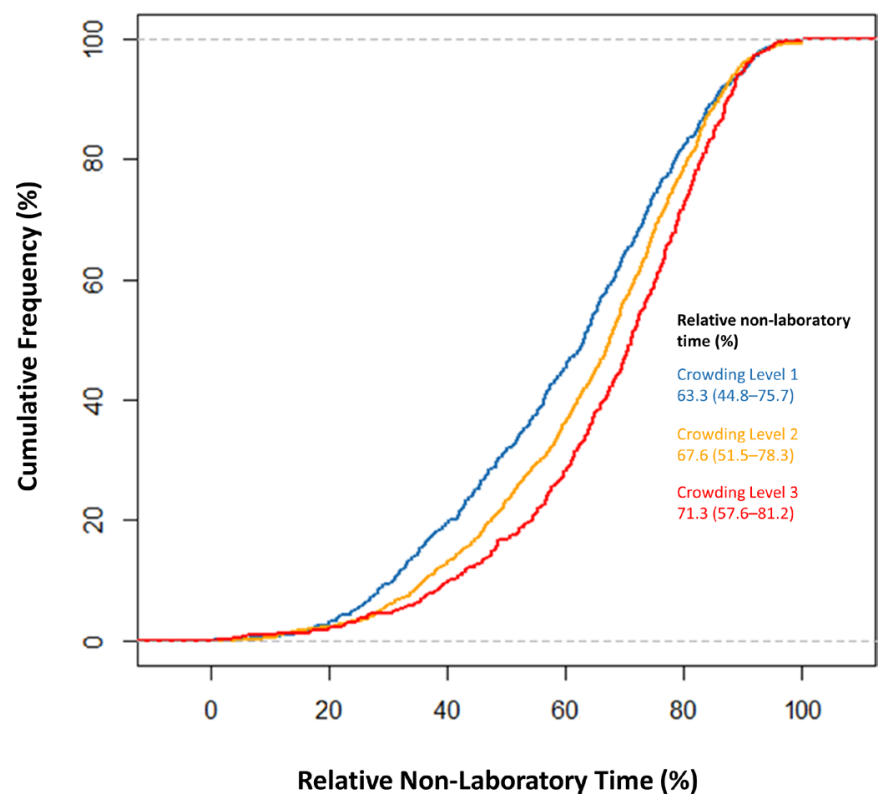

Figure 4 Cumulative distribution of the relative nonlaboratory time (\%) by crowding level: normal (blue), high (yellow) and very high crowding (red). time for additional diagnostic work-up and drafting of the discharge or referral report. The cumulative distributions of the absolute and relative non-laboratory time are shown on figures 3 and 4, and multiple regression analyses in online supplemental tables S1 and S2.

Crowding had an effect on utilisation rates of ESC recommended diagnostic protocols $(\mathrm{p}=0.009$ for trend). Rates of delayed blood draws exceeding the upper $30 \mathrm{~min}$ tolerance bound increased significantly from $7.5 \%$ to $11.7 \%$ with the highest crowding level, when the $0 / 3$ hours algorithm was used $(\mathrm{p}<0.001)$. Crowding was also associated with increasing rates of additional troponin testing after completed diagnostic testing. The proportion of patients with additional hsTnT beyond diagnostic set increased significantly in higher crowding from $29.6 \%$ to $37.7 \%$ ( $\mathrm{p}=0.001)$.

\section{All-cause mortality and crowding}

In total, there were 143 deaths (Kaplan-Meier estimates for 30-day mortality $1.8 \%$ and for 1-year mortality $5.3 \%$ ). Of them, 36 deaths were in the subgroup of patients treated in low crowding setting, 80 in high crowding, and 27 in very high crowding (Kaplan-Meier estimates for 30 -day mortality $1.8 \%, 1.8 \%$ and $2.0 \%$, respectively; and for 1-year mortality $5.4 \%, 5.1 \%$ and $5.8 \%$, respectively). Higher crowding levels were not significantly associated with higher all-cause mortality rates (figure 5, log-rank $\mathrm{p}=0.9$ ).

In a Cox proportional hazards model, higher admission hsTnT and $\mathrm{C}$ reactive protein, higher age, lower systolic blood pressure, higher heart rate and prior MI were independently associated with mortality, but neither crowding level nor study period, nor chronic kidney disease (table 5).

\section{DISCUSSION}

In this study, we provide important information on the effects of increasing level of crowding from a single-centre observational study that aimed to evaluate the effects of implementation of fast protocols on safety of discharge in low-risk patients with suspected ACS, and the effects on length of ED stay, blood sampling intervals and laboratory TAT and discharge rates. In agreement with previously suggested criteria for crowding, ${ }^{22-24}$ our definition used criteria that reflect (1) the mismatch between numbers of patients visiting the ED and monitoring capacities and (2) effective physician time per case. The criteria were adapted to the staff and monitoring capacities in our ED. Accordingly, we defined three level of crowding (normal, high and very high) and plotted 30-day and 1-year outcomes and key performance parameters with available electronic time-stamps across crowding levels.

We report seven important findings. First, crowding had no negative effect on 30-day or 1-year mortality. Second, crowding had a significant effect on the length of ED stay, regardless whether patients were admitted or discharged home. Third, while the result was not 
A

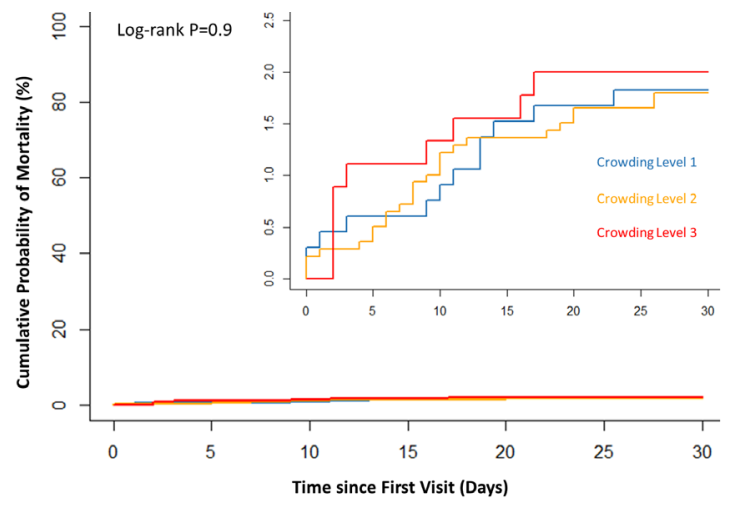

No. at Risk

Crowding Level 1, normal 662 Crowding Level 2, high 1409

Crowding Level 3 , very high 454

\section{2 \\ 1380}

651

1371

646

1366

645

1364

644

1362

644

$\begin{array}{lllll}444 & 443 & 441 & 441\end{array}$

B

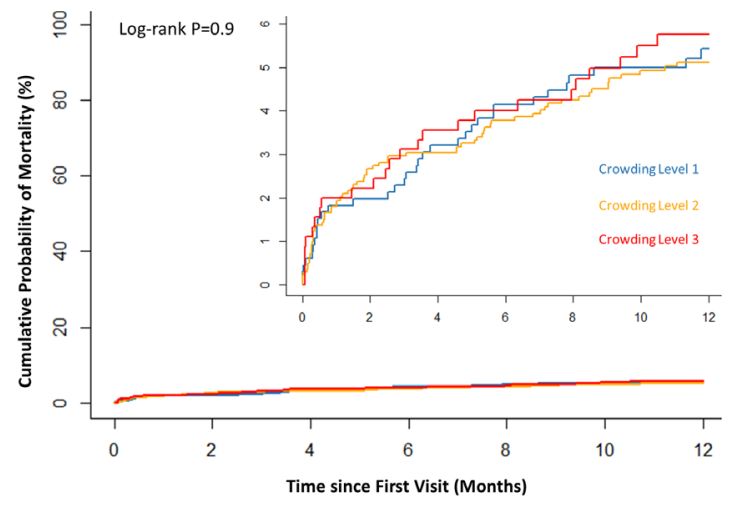

No. at Risk

Crowding Level 1 , normal 662

Crowding Level 2, high $\quad 1409$

Crowding Level 3 , very high 454
643

1346

440
626 1317

429

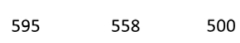

1261

Figure 5 Kaplan-Meier estimates of 30 days $(A)$ and 1year (B) mortality in patients with suspected acute coronary syndrome by crowding level in the emergency department: normal (blue), high (yellow) and very high crowding (red).

statistically significant, we observed higher discharge rates from ED during episodes of very high crowding $(62.8 \%)$, as compared with normal crowding $(58.9 \%)(\mathrm{p}=0.080)$. Our data do not allow us to provide an exact underlying reason. However, it is tempting to speculate that higher discharge rates may indirectly reflect shortage of hospital bed capacities. Fourth, crowding was associated with significantly lower utilisation of fast diagnostic protocols recommended by 2015 ESC guidelines. ${ }^{11}$ Fifth, increasing crowding prolonged the sampling interval between the first and second blood draw by a median of $9 \mathrm{~min}$, and the median laboratory TAT by $4 \mathrm{~min}$. Sixth, length of ED stay was disproportionately long despite the use of fast protocols. However, the small but significant prolongation of blood sampling intervals and blood processing times does not explain this excessive prolongation of length of ED stay, suggesting additional delays due to other processes, such as delays for additional diagnostic workup, or related to the process of discharge. Accordingly, we observed a disproportionate increase of time delays that were not related to the diagnostic biomarker algorithm itself, but rather with time delays associated with diagnostic workup, drafting of referral/discharge report and/or other unspecified processes. In line with our hypothesis, Ambavane $e t a l^{21}$ reported in an economic evaluation of the High Sensitivity Cardiac Troponin $\mathrm{T}$ Assay for Rapid Rule-out of Acute Myocardial Infarction (TRAPID AMI) trial $^{12}$ that workup for differential diagnoses and report drafting consumed additional 2.5 hours after rule-out process, thus contributing considerably to the short overall length of ED stay of 4 hours using the ESC 0/1 hour algorithm. ${ }^{21}$ Seventh, crowding was associated with increased numbers of avoidable additional hsTnT measurements and thus increased costs for assays.

Our findings come timely as the Centers for Medicare \& Medicaid Services have mandated the collection and public reporting of ED crowding measures for both admitted and discharged patients as quality-of-care markers since January $2014^{26}$ For discharged patients, this includes waiting time and LOS (Medicare). The three measures most frequently linked to the quality of care include the number of patients in the waiting room, ED occupancy (percentage of overall ED beds filled), and the number of admitted patients in the ED awaiting inpatient beds. $^{22-24}$

\section{Previous findings on ED crowding and outcomes}

A review article $^{1}$ identified several studies, in which crowding in the ED was associated with prolonged process times, such as time to administration of analgesics or antibiotics. ${ }^{427}$ Another three studies were focused on the association between crowding in the ED and mortality. ${ }^{5} 2829$ While most findings show an association between ED crowding and mortality in patients admitted to the hospital, there is less evidence for the population of patients discharged from the ED. ${ }^{1}$ In a populationbased cohort study from Canada, the average length of ED stay for the shift was associated with higher short-term mortality and hospitalisation rates in patients discharged directly from the ED. ${ }^{5}$ In a retrospective analysis of patients admitted with chest pain, Pines et $a l^{30}$ found an association between several measures of ED crowding and adverse cardiovascular outcomes (defined as MI, heart failure, hypotension, dysrhythmias and cardiac arrest) - both in ACS-related and non-ACS-related chest pain. However, single measures of ED crowding were used and the findings were not supported by similar studies. Gabayan $e t a l^{31}$ found that only evaluation time and total LOS were associated with a short-term admission after discharge. No association was found between the measures of crowding in the ED and mortality, both for patients hospitalised and after discharge. Ko $e t a l^{32}$ reported that increasing volumes of chest pain visits were associated with a relative decrease in the odds of mortality or hospitalisations for ACS, a finding that was even more pronounced among patients at higher risk. These differences in results regarding crowding could be explained by the different healthcare systems and study cohorts. Another possible explanation is the difference in adjustment for comorbidities and disease severity. In preliminary analyses using 
Table 5 Results of Cox proportional hazards regression for predictors of mortality

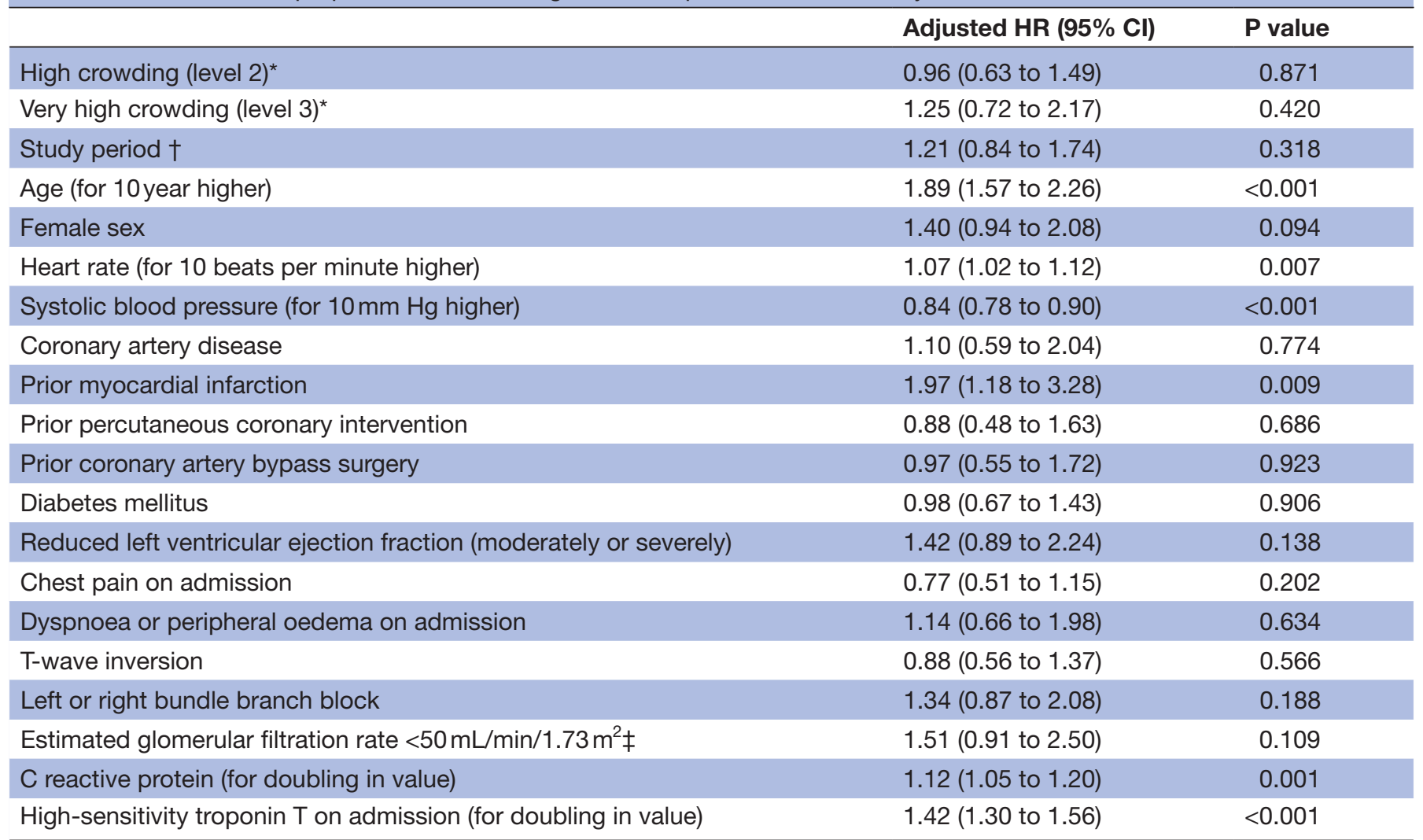

${ }^{*}$ For the comparison with normal crowding (level 1).

†For the comparison of study period two with study period 1.

‡By modification of diet in renal disease equation.

models fitted on a small subset of variables (ie, only age and sex), and no covariates representing disease severity and comorbidities, the rates of admission and mortality was higher. However, after adjustment for more clinical variables representing severity of disease, associations were shown to be weaker. It may be speculated that length of ED stay may be confounded by unmeasured variables, including disease severity, and not independently associated with adverse outcomes. ${ }^{31}$

\section{Limitations}

There are several limitations that have to be discussed. First, the present evaluation was executed in a single wellorganised ED led by a cardiologist in a tertiary referral centre with long-term implementation of hsTnT and fast diagnostic algorithms. Access to central laboratory services may vary largely affecting protocol-related time delays to a considerably higher degree in other hospital settings. Second, our findings were obtained in patients with suspected ACS and excluded patients who were ineligible for the main study that focused on the implementation of fast diagnostic protocols. Therefore, our findings cannot be extrapolated to other settings such as general EDs. Third, the absolute and relative delays of non-laboratory-related processes is hospital-specific and also depends on the intensity of workup provided beyond the biomarker based protocol, such as additional imaging and/or stress testing. Fourth, not all variables that might account for the overall non-laboratory time excess such as ambulance offload times or times to nurse triage were recorded. Therefore, and because laboratory related delays were minimal at our institution, an underestimation of the effect of crowding on the overall length of ED stay is anticipated. Fifth, additional factors other than crowding may also affect length of ED stay, especially additional diagnostic workup including predischarge stress tests or cardiac imaging.

Another limitation is related to the definition of crowding. Our definition is not established and does not claim to include all previously proposed measures, but is based on two basic assumptions that are believed to describe overcrowding and have the advantage that they can be quantified. These two elements consist of (1) the mismatch between bed capacities and number of patients admitted, and (2) the adjusted times that a physician spends on a case to reflect work load. While the latter is independent of the hospital setting, crowding level by mismatch of monitoring capacities has to be calculated for a hospital individually using the same assumptions as in our definition. 


\section{CONCLUSION}

In conclusion, crowding in the ED might affect both protocol-related times but also laboratory-independent time delays to various degrees, thus diminishing some positive effects of fast diagnostic protocols in suspected ACS. Our findings demonstrate that even with optimisation of laboratory-dependent processes, the workflow in the ED has to be optimised, so as to achieve the most advantages of earlier diagnosis.

Acknowledgements We thank the staff of the emergency department and express our gratitude to study nurses Heidi Deigentasch, Melanie Hütter and Elisabeth Mertz.

Contributors KMS, MB, HH and EG were involved in the conception and design of the study, the acquisition, analysis and interpretation of data, drafted the manuscript, approved the final version to be published, are accountable for all aspects of the work. MM-H, MV and HAK were involved in the interpretation of data, critically revised the manuscript for important intellectual content, approved the final version to be published and agreed to be accountable for all aspects of the work. The corresponding author attests that all listed authors meet authorship criteria and that no others meeting the criteria have been omitted.

Funding This work was supported by a research grant from Roche Diagnostics International (no. 7100034680).

Competing interests MB reports grants and non-financial support from AstraZeneca, non-financial support from Thermo Fisher. MM-H reports grants and speaker honoraria from Roche Diagnostics; grants and non-financial support from BRAHMS Thermo Scientific. HAK received honoraria for lecturers from Roche Diagnostics, AstraZeneca, Bayer Vital, Daiichi-Sankyo, and held a patent on $\mathrm{CTnT}$ that has expired. EG received honoraria for lectures from Roche Diagnostics, AstraZeneca, Bayer Vital, Daiichi-Sankyo, Eli Lilly Deutschland. He serves as a consultant for Roche Diagnostics, BRAHMS Thermo Fisher, Boehringer Ingelheim, and has received research funding from BRAHMS Thermo Fisher, Roche Diagnostics, Bayer Vital and Daiichi Sankyo.

Patient consent for publication Not required.

Ethics approval The study was approved by the Ethics Committee of the Medical Faculty of Heidelberg and was conducted according to ethical principles stated in the Declaration of Helsinki. Ethics Committee of the Medical Faculty of Heidelberg (S-313/2017)

Provenance and peer review Not commissioned; externally peer reviewed.

Data availability statement No data are available. The present study evaluated data collected retrospectively from routine care of consecutive patients, admitted to the emergency department. To share patient-level data, permission by each patient is necessary. Since this is not possible, we are not allowed to share patient level-data.

Open access This is an open access article distributed in accordance with the Creative Commons Attribution Non Commercial (CC BY-NC 4.0) license, which permits others to distribute, remix, adapt, build upon this work non-commercially, and license their derivative works on different terms, provided the original work is properly cited, appropriate credit is given, any changes made indicated, and the use is non-commercial. See: http://creativecommons.org/licenses/by-nc/4.0/.

ORCID iD

Evangelos Giannitsis http://orcid.org/0000-0002-1025-2872

\section{REFERENCES}

1 Carter EJ, Pouch SM, Larson EL. The relationship between emergency department crowding and patient outcomes: a systematic review. J Nurs Scholarsh 2014;46:106-15.

2 Stang AS, Crotts J, Johnson DW, et al. Crowding measures associated with the quality of emergency department care: a systematic review. Acad Emerg Med 2015;22:643-56.

3 Morley C, Unwin M, Peterson GM, et al. Emergency department crowding: a systematic review of causes, consequences and solutions. PLoS One 2018;13:e0203316.

4 Bernstein SL, Aronsky D, Duseja R, et al. The effect of emergency department crowding on clinically oriented outcomes. Acad Emerg Med 2009;16:1-10.
5 Guttmann A, Schull MJ, Vermeulen MJ, et al. Association between waiting times and short term mortality and hospital admission after departure from emergency department: population based cohort study from Ontario, Canada. BMJ 2011;342:d2983.

6 Rui P, Kang K, Ashman JJ. National Hospital ambulatory medical care survey: 2016 emergency department summary tables, 2016. Available: https://www.cdc.gov/nchs/data/nhamcs/web tables/ 2016_ed_web_tables.pdf [Accessed 10 Jan 2020].

7 Fanaroff AC, Rymer JA, Goldstein SA, et al. Does this patient with chest pain have acute coronary syndrome?: the rational clinical examination systematic review. JAMA 2015;314:1955-65.

8 Goodacre S, Thokala P, Carroll C, et al. Systematic review, metaanalysis and economic modelling of diagnostic strategies for suspected acute coronary syndrome. Health Technol Assess 2013;17:1-188. v-vi.

9 Nuffield Trust \& The Health Foundation. Potentially preventable emergency hospital admissions, 2019. Available: https://www. nuffieldtrust.org.uk/resource/potentially-preventable-emergencyhospital-admissions [Accessed $18 \mathrm{Jul} 2020]$.

10 Eichelberger C, Patel A, Ding Z, et al. Emergency department visits and subsequent hospital admission trends for patients with chest pain and a history of coronary artery disease. Cardiol Ther 2020;9:153-65.

11 Roffi M, Patrono C, Collet J-P, et al. 2015 ESC guidelines for the management of acute coronary syndromes in patients presenting without persistent ST-segment elevation: Task force for the management of acute coronary syndromes in patients presenting without persistent ST-segment elevation of the European Society of cardiology (ESC). Eur Heart J 2016;37:267-315.

12 Mueller C, Giannitsis E, Christ M, et al. Multicenter evaluation of a 0-Hour/1-Hour algorithm in the diagnosis of myocardial infarction with high-sensitivity cardiac troponin T. Ann Emerg Med 2016;68:76-87.

13 Neumann JT, Sörensen NA, Schwemer T, et al. Diagnosis of myocardial infarction using a high-sensitivity troponin I 1-hour algorithm. JAMA Cardiol 2016;1:397-404.

14 Mokhtari A, Borna C, Gilje P, et al. A 1-h Combination Algorithm Allows Fast Rule-Out and Rule-In of Major Adverse Cardiac Events. $J$ Am Coll Cardiol 2016;67:1531-40.

15 Than M, Aldous S, Lord SJ, et al. A 2-hour diagnostic protocol for possible cardiac chest pain in the emergency department: a randomized clinical trial. JAMA Intern Med 2014;174:51-8.

16 Möckel M, Searle J, Hamm C, et al. Early discharge using single cardiac troponin and copeptin testing in patients with suspected acute coronary syndrome (ACS): a randomized, controlled clinica process study. Eur Heart $J$ 2015;36:369-76.

17 Carlton EW, Ingram J, Taylor H, et al. Limit of detection of troponin discharge strategy versus usual care: randomised controlled trial. Heart 2020. doi:10.1136/heartjnl-2020-316692. [Epub ahead of print: 05 May 2020].

18 Mahler SA, Riley RF, Hiestand BC, et al. The heart pathway randomized trial: identifying emergency department patients with acute chest pain for early discharge. Circ Cardiovasc Qual Outcomes 2015;8:195-203.

19 Twerenbold R, Jaeger C, Rubini Gimenez M, et al. Impact of highsensitivity cardiac troponin on use of coronary angiography, cardiac stress testing, and time to discharge in suspected acute myocardial infarction. Eur Heart J 2016;37:3324-32.

20 Cheng Q, Greenslade JH, Parsonage WA, et al. Change to costs and lengths of stay in the emergency department and the Brisbane protocol: an observational study. BMJ Open 2016;6:e009746.

21 Ambavane A, Lindahl B, Giannitsis E, et al. Economic evaluation of the one-hour rule-out and rule-in algorithm for acute myocardial infarction using the high-sensitivity cardiac troponin T assay in the emergency department. PLoS One 2017;12:e0187662.

22 Hwang U, McCarthy ML, Aronsky D, et al. Measures of crowding in the emergency department: a systematic review. Acad Emerg Med 2011;18:527-38

23 Ospina MB, Bond K, Schull M, et al. Key indicators of overcrowding in Canadian emergency departments: a Delphi study. CJEM 2007:9:339-46.

24 Solberg LI, Asplin BR, Weinick RM, et al. Emergency department crowding: consensus development of potential measures. Ann Emerg Med 2003;42:824-34.

25 Stoyanov KM, Hund H, Biener M, et al. RAPID-CPU: a prospective study on implementation of the ESC 0/1-hour algorithm and safety of discharge after rule-out of myocardial infarction. Eur Heart $J$ Acute Cardiovasc Care 2020;9:39-51.

26 Medicare. Hospital compare measures, 2019. Available: https:// www.cms.gov/Medicare/Quality-Initiatives-Patient-AssessmentInstruments/HospitalQualitylnits/HospitalProcessOfCareMeasures [Accessed 10 Jan 2020]. 
27 Johnson KD, Winkelman C. The effect of emergency department crowding on patient outcomes: a literature review. Adv Emerg Nurs $\mathrm{J}$ 2011;33:39-54.

28 Cha WC, Shin SD, Cho JS, et al. The association between crowding and mortality in admitted pediatric patients from mixed adultpediatric emergency departments in Korea. Pediatr Emerg Care 2011;27:1136-41.

29 Richardson DB. Increase in patient mortality at 10 days associated with emergency department overcrowding. Med J Aust 2006;184:213-6.
30 Pines JM, Pollack CV, Diercks DB, et al. The association between emergency department crowding and adverse cardiovascular outcomes in patients with chest pain. Acad Emerg Med 2009;16:617-25.

31 Gabayan GZ, Derose SF, Chiu VY, et al. Emergency department crowding and outcomes after emergency department discharge. Ann Emerg Med 2015;66:483-92.

32 Ko DT, Dattani ND, Austin PC, et al. Emergency department volume and outcomes for patients after chest pain assessment. Circ Cardiovasc Qual Outcomes 2018;11:e004683. 